\title{
Stability of Sliding Frictional Surfaces with Varying Normal Force ${ }^{1}$
}

\author{
Pierre E. Dupont Deepak Bapna \\ Aerospace and Mechanical Engineering \\ Boston University \\ Boston MA 02215
}

December 27, 1993

\begin{abstract}
This paper presents the stability analysis of a single degree of freedom elastic system following a rate- and state-dependent friction law. Normal force is assumed to depend on the displacement, velocity and acceleration of the sliding interface. The history dependence of friction on normal force is included in the analysis. It is shown that to achieve steady sliding, system stiffness must exceed a critical value which depends on the expression for normal force. A system in which normal force depends on spring displacement is analyzed in detail. These results indicate that the functional dependence of normal force on system state can have a significant effect on the stability of low-velocity motion.
\end{abstract}

\section{Introduction}

Stick-slip motion, characterized by intermittent sticking and slipping of bodies in sliding contact, is observed in many mechanical systems. In some systems, such as turbomachinery and large flexible space structures, the frictional damping inherent in this motion is advantageous for passive control of vibrations (Anderson and Ferri 1990). More often, it is a detrimental effect encountered when the desired system motion involves low velocities. It occurs in machine tools, tracking mechanisms, robots under position or force control and even, apparently, during earthquakes. For these systems, it is desirable to predict whether or not stick slip will occur. Except for the last example, this type of analysis may facilitate redesign of the mechanism and controller so that stick slip can be avoided.

The stability of constant-velocity sliding motion is the topic of this paper. This topic can be studied by either considering the stability of the constant-velocity equilibrium point or the instability of stick-slip limit cycles. The former approach will be taken here. Investigations

\footnotetext{
${ }^{1}$ Presented in the Symposium on Friction-induced Vibration, Chatter, Squeal and Chaos at the ASME Winter Annual Meeting, Anaheim, CA, November, 1992. This work was supported in part by the National Science Foundation under Grants MSS-9112049 and MSS-9302190.
} 


\section{Nomenclature}

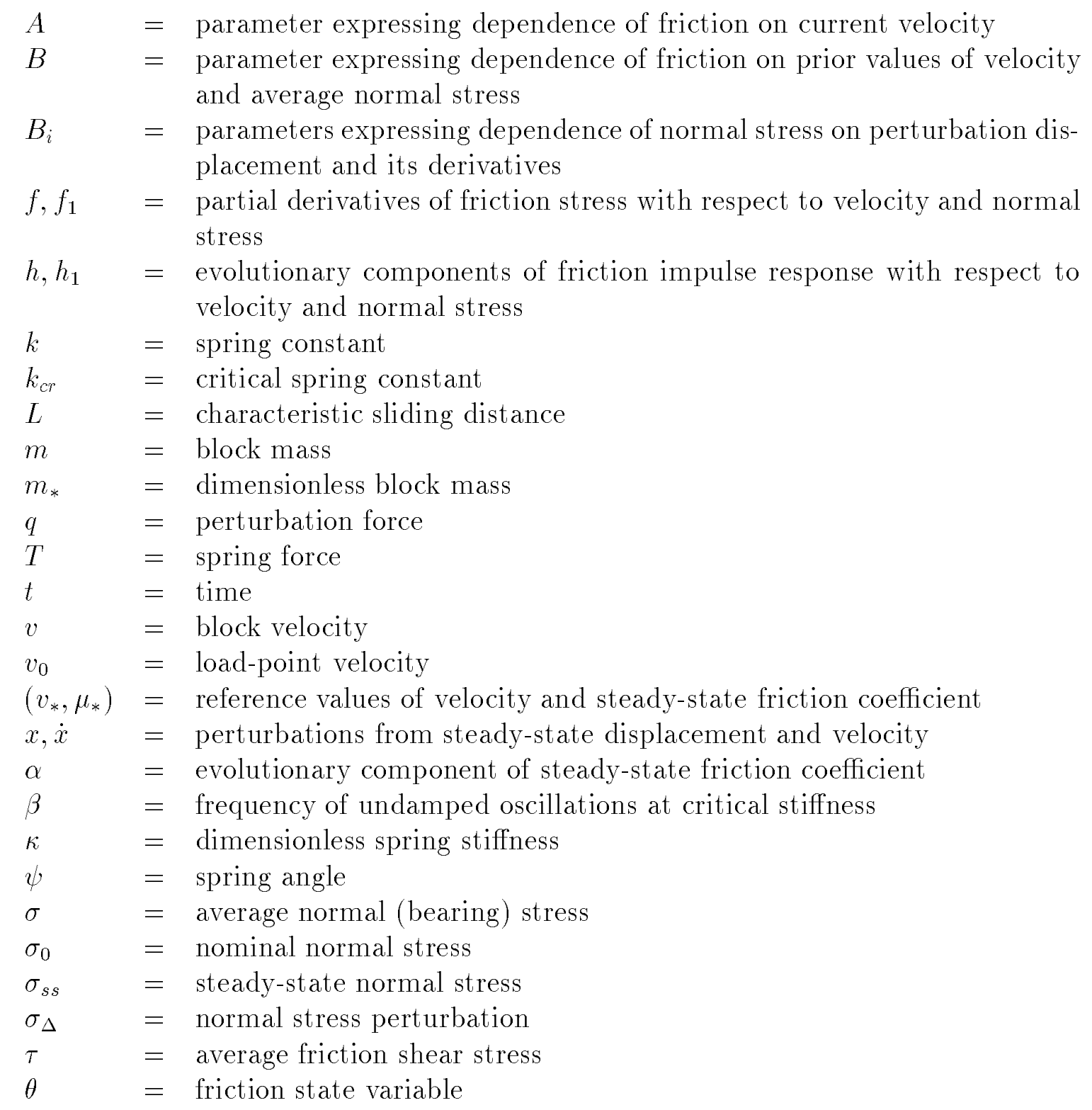


of equilibrium-point stability have been carried out by Rice and Ruina (1983) and Dupont (1994). Note that both the equilibrium point and the stick-slip limit cycle of a system may be stable simultaneously. In this case, large perturbations may move the system from the domain of attraction of the equilibrium point to that of the limit cycle. Also, when starting from rest, it may be necessary to perturb the system away from the limit cycle to induce stable sliding.

A factor which has received little attention in the literature is the effect of normal force. While most analyses assume it to be constant, normal force is often a function of the system state. For example, in any drive mechanism, whether composed of screws, gears or cams, the normal force changes during motion. Thus, it is not clear that stability results obtained for constant normal force can be directly applied to many systems.

Anderson and Ferri (1990) and Bindemann and Ferri (1992) have considered the effect of varying normal force on friction dampers. These systems can be viewed as position regulators subject to a harmonic disturbance. The equilibrium point corresponds to zero velocity. Any motion is due to application of the disturbance force.

In this paper, the stability of constant-velocity slipping is considered. The paper is arranged as follows. In the next section, friction modeling is discussed and state variable friction laws are introduced. In section 3 , a linearized analysis of a sliding system subject to perturbations about a constant sliding velocity is presented. The result of this analysis is a critical value of stiffness. Systems with stiffnesses greater than this critical value are stable in steady sliding motion. In this analysis, the normal force can depend on the relative slip displacement, velocity and acceleration. To illustrate these results, a specific example is provided in section 4 for which normal force depends linearly on spring dispacement. Both quasistatic and inertial models are considered with numerical simulation used for validation and comparison. Conclusions appear in section 5 .

\section{$2 \quad$ Friction Modeling}

It has long been known that friction is not determined by current velocity alone; it also depends on the history of motion. The history dependence of friction has been studied extensively by the rock mechanics community (Ruina 1983; Rice and Ruina 1983; Gu et al. 1984; Dieterich 1991; Linker and Dieterich 1992). Interest in rock friction stems from the hypothesis that earthquakes are fault-line stick-slip events. In order to approximate fault-line slip, experiments have been conducted at moderate normal stresses (e.g., $5 \mathrm{MPa}$, computed using apparent area of contact) and very low velocities (e.g., $10^{-2}-10^{2} \mu \mathrm{m} / \mathrm{sec}$ ) with conformal contacts.

Based on these experiments, state variable friction laws have been proposed. While these laws have been developed for rocks, their velocity effects have recently been observed for a range of materials (Dieterich 1991; Dupont and Dunlap 1993). These include lubricated 
steel on steel, teflon on steel, glass, plastic and wood. State variable models, as developed by Dieterich, Ruina and Linker, are described below.

\subsection{State Variable Friction Laws}

Dieterich $(1972,1979)$ and Ruina (1983) have performed numerous friction experiments on rocks in which they have observed a fading memory dependence of friction on slip history. They argue that for constant normal load, friction can be described by the current slip velocity and by a set of state variables which represent the surface's memory of previous sliding. In general, these are weighted averages of some function of the recent sliding velocity.

Several researchers have studied the transient behavior of friction in response to changes in normal load. These include Hobbs and Brady (1985) and Lockner et al. (1986). Allobserved that a sudden increase (decrease) in normal load causes a sudden increase (decrease) in friction and an evolutionary increase (decrease) in friction to a new steady-state level as sliding proceeds.

Linker and Dieterich (1992) carried out a detailed investigation using a double-shear apparatus with $5 \mathrm{~cm} \times 5 \mathrm{~cm}$ blocks of westerly granite. They performed tests involving step changes and pulses in normal load during constant-velocity sliding. A reference normal stress of $5 \mathrm{MPa}$ and load-point velocity of $1 \mu \mathrm{m} / \mathrm{sec}$ were $u$ sed.

They confirmed the observations that a sudden change in normal load causes a sudden change in the friction followed by an evolutionary change. To incorporate the observed effects into a state variable model, they made the following three assumptions:

1. Changes in normal stress, $\sigma$, result in changes in state, $\theta$.

2. The changes in shear stress, $\tau$, that result from changes in $\sigma$ and $v$ can be expressed by the same state variable, $\theta$.

3. A sudden change in $\sigma$ results in a sudden change in the state variable. This change is symmetric with respect to increases and decreases in $\sigma$.

Linker and Dieterich proposed the following model.

$$
\begin{aligned}
\tau & =\sigma\left[\mu_{*}+A \ln \left(\frac{v}{v_{*}}\right)+B \theta\right] \\
\dot{\theta} & =-\frac{v}{L}\left[\ln \left(\frac{v}{v_{*}}\right)+\theta\right]-\frac{\alpha}{B \sigma} \dot{\sigma}
\end{aligned}
$$

In this equation, $\mu_{*}$ is the steady state coefficient of friction at the reference velocity, $v_{*}$. The parameter $\alpha$ is an empirical constant and is a measure of the evolutionary component 


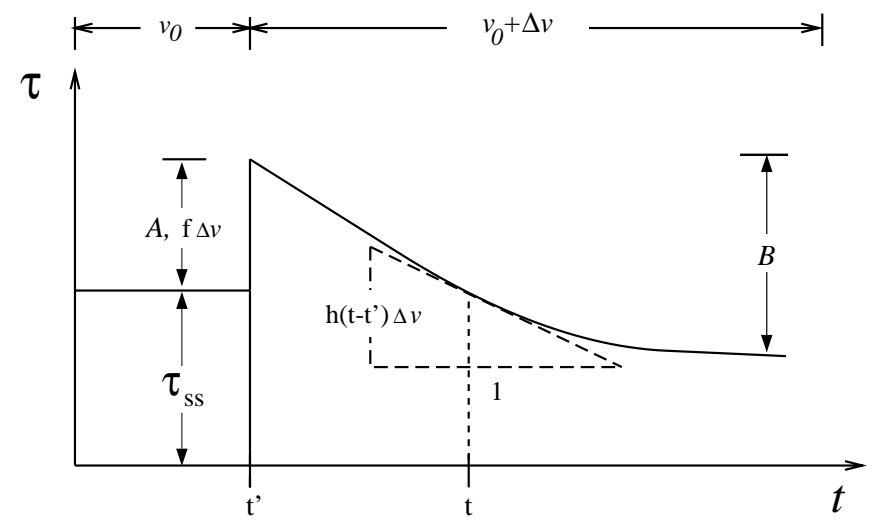

Figure 1: Friction response to a step change in velocity. ( $f$ and $h$ are used in the stability analysis to follow.)

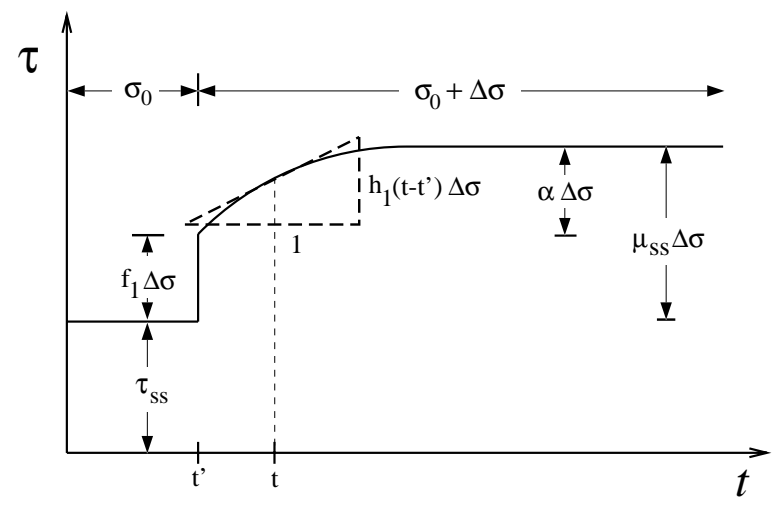

Figure 2: Friction response to a step change in normal stress. ( $f_{1}$ and $h_{1}$ are used in the stability analysis to follow.)

of the change in friction stress due to a change in normal stress. This model is used in the analyses which follow.

Since the state variable friction laws were developed to model interfacial stresses in geologic faults, they are written in terms of normal stress, $\sigma$, and friction shear stress, $\tau$. This notation will be used here for consistency. However, it should be noted that these are average quantities which refer to the normal and friction forces divided by apparent area of contact.

Figures 1 and 2 depict the response of (1) to sudden steps in sliding velocity, $\Delta v$, and normal stress, $\Delta \sigma$, respectively. This behavior corresponds to experimental observations. In Figure 1, the changes in friction stress denoted by $A$ and $B$ correspond to the velocity step, $\Delta v=(e-1) v_{0}$ where $e=2.71828 \ldots$

For the case of constant normal stress, Dieterich (1979) provides a physical interpretation of the state variable. He proposes that $\theta$ is proportional to the apparent age of the asperity 


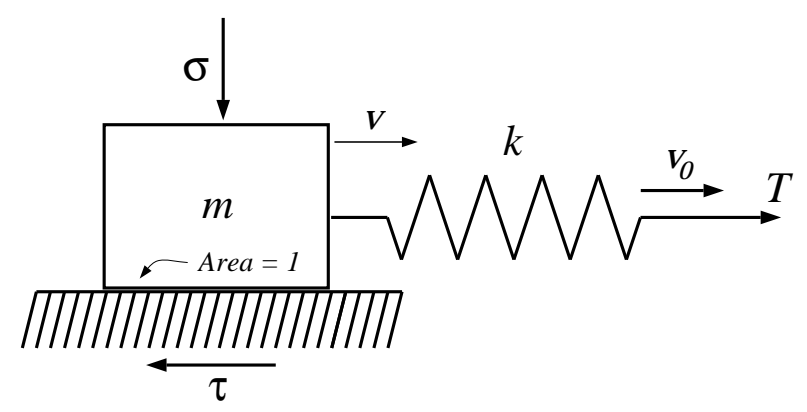

Figure 3: Spring-mass model.

junctions. When normal stress can vary, Linker and Dieterich (1992) propose that $\theta$ is related to the fraction of the contact area associated with time-dependent creep.

\section{Critical Stiffness}

It has been observed experimentally that as the stiffness of a system is increased, the stickslip amplitude decreases. It is often the case that above a certain stiffness, stick-slip motion changes to quasi-steady sliding (Rabinowicz 1958; Rice and Ruina 1983). It is important to be able to predict this stiffness, called the critical stiffness, which will ensure stable sliding. Friction laws which depend only on the current slip rate cannot be used to predict the critical stiffness since they indicate that only by applying sufficient damping can stick-slip be eliminated. Models which include frictional lag must be used to predict steady motion for stiff systems (Armstrong-Hélouvry 1991; Rice and Ruina 1983; Dupont 1994).

Except for (Dieterich and Linker 1992), the effect of varying normal stress and the associated frictional lag on critical stiffness has received little attention. In this section, this stiffness is

derived for a single degree of freedom elastic system for which normal stress depends on the system state and its derivatives.

The system is represented by a slider attached to a spring as shown in Figure 3 . The free end of the spring is constrained to move at velocity, $v_{0}$, called the load-point velocity.

Let

$$
v(t)=v_{0}+\dot{x}(t)
$$

In this equation, $x$ can be interpreted as shortening of the spring from its steady-state length.

Given unit base area, the force exerted by the spring can be written as

$$
T=\tau_{s s}-k x
$$

where $\tau_{s s}$ is the steady-state friction stress. 
The equation of motion is therefore

$$
m \ddot{x}=\tau_{s s}-k x-\tau+q
$$

where $q$ is a disturbance force.

In order to analyze the stability of the system, small perturbations about the load-point velocity, $v_{0}$, and steady-state normal stress, $\sigma_{s s}$, are considered.

$$
\begin{aligned}
& v(t)=v_{0}+\dot{x}(t), \quad\left|\frac{\dot{x}(t)}{v_{0}}\right|<<1 \\
& \sigma(t)=\sigma_{s s}+\sigma_{\Delta}(t), \quad\left|\frac{\sigma_{\Delta}(t)}{\sigma_{s s}}\right|<<1
\end{aligned}
$$

The impulse response is used to represent the linearized behavior of $\tau(t)$ about the equilibrium point $\left(v_{0}, \sigma_{s s}\right)$. A graphical representation of this appears in Figures 1 and 2 . The stress, $\tau(t)$, can be expressed as

$$
\tau(t)=\tau_{s s}\left(v_{0}, \sigma_{s s}\right)+f \dot{x}(t)-\int_{0}^{t} h\left(t-t^{\prime}\right) \dot{x}\left(t^{\prime}\right) d t^{\prime}+f_{1} \sigma_{\Delta}(t)+\int_{0}^{t} h_{1}\left(t-t^{\prime}\right) \sigma_{\Delta}\left(t^{\prime}\right) d t^{\prime}
$$

where $\theta$ is the state variable and all of the following derivatives are evaluated at the equilibrium point.

$$
\begin{aligned}
\frac{\partial \tau}{\partial v} & =f>0 \\
\frac{d \tau_{s s}}{d v} & =f-\int_{0}^{\infty} h(t) d t<0 \\
\frac{\partial \tau}{\partial \sigma} & =f_{1}>0 \\
\frac{d \tau_{s s}}{d \sigma} & =f_{1}+\int_{0}^{\infty} h_{1}(t) d t=\mu_{s s}>0
\end{aligned}
$$

where $\mu_{s s}$ is the steady-state coefficient of friction.

The linearized equation of motion can now be written as

$$
m \ddot{x}(t)+f \dot{x}(t)-\int_{0}^{t} h\left(t-t^{\prime}\right) \dot{x}\left(t^{\prime}\right) d t^{\prime}+f_{1} \sigma_{\Delta}(t)+\int_{0}^{t} h_{1}\left(t-t^{\prime}\right) \sigma_{\Delta}\left(t^{\prime}\right) d t^{\prime}+k x(t)=q(t)
$$

Taking the Laplace transform of (10) and rearranging terms gives

$$
\left\{m s^{2}+\{f-\hat{h}(s)\} s+k\right\} \hat{x}(s)+\left\{f_{1}+\hat{h}_{1}(s)\right\} \hat{\sigma}^{*}(s)=\hat{q}(s)
$$

In this equation, while $x$ is a system variable and is defined by $(3), \sigma$ is a dependent variable which must be specified in terms of the system variables. 
A general equation for normal stress expressing a linearized dependence on acceleration, velocity and displacement, respectively, is given by

$$
\hat{\sigma}^{*}(s)=\left(B_{0} s^{2}+B_{1} s+k B_{2}+B_{3}\right) \hat{x}(s)+B_{4}
$$

In this expression $B_{0}$ and $B_{1}$ express the normal-stress dependence on acceleration and velocity, respectively. The parameter $B_{2}$ represents a dependence on displacement coupled through stiffness while $B_{3}$ represents a direct dependence on displacement. Step changes in normal stress at $t=0$ can be represented by $B_{4}$.

For stable sliding, the roots of the characteristic equation should lie in the left half of the complex plane. Substituting $s= \pm \iota \beta$ into the characteristic equation, we wish to solve for the critical value of spring stiffness at which the roots of the characteristic equation pass through the imaginary axis into the right half plane. For the exponential decays depicted in Figure 1 and $2, h(t)$ and $h_{1}(t)$ can be represented by

$$
\begin{array}{rlrl}
h(t) & =(1+\lambda) r f e^{-r t}, & \lambda>0 \\
h_{1}(t) & =\left(\lambda_{1}-1\right) r f_{1} e^{-r t}, \quad \lambda_{1}>1
\end{array}
$$

Here $1 / r$ represents the characteristic time of the decay processes which, according to Dieterich and Linker (1992), is the same for velocity steps and normal stress steps. Also,

$$
\begin{aligned}
& \frac{d \tau_{s s}}{d v}=-\lambda f \\
& \frac{d \tau_{s s}}{d \sigma}=\lambda_{1} f_{1}=\mu_{s s}
\end{aligned}
$$

The following equations are obtained by combining (11)-(14), evaluating at $s=i \beta$ and separating the result into real and imaginary parts.

$$
\begin{gathered}
-\beta^{4}\left(m+B_{0} f_{1}\right)+\beta^{2}\left\{-r^{2}\left(m+B_{0} \lambda_{1} f_{1}\right)+k+f_{1}\left(k B_{2}+B_{3}\right)\right. \\
\left.+B_{1}\left(\lambda_{1}-1\right) r f_{1}-(1+\lambda) r f\right\}+r^{2}\left\{k+f_{1} \lambda_{1}\left(k B_{2}+B_{3}\right)\right\}=0 \\
\beta^{2}=\frac{\left(k B_{2}+B_{3}\right)\left(\lambda_{1}-1\right) r f_{1}+r^{2}\left(f \lambda-f_{1} B_{1} \lambda_{1}\right)}{B_{0}\left(\lambda_{1}-1\right) r f_{1}+\left(f+B_{1} f_{1}\right)}
\end{gathered}
$$

Substituting (18) into (17) yields a quadratic equation in $k$ with roots which can be found with some effort. These are

$$
k_{c r_{1}}=-\frac{f_{1}\left(\lambda_{1}-1\right)\left(B_{3}-B_{1} r+B_{0} r^{2}\right)+f r(\lambda+1)}{B_{2} f_{1}\left(\lambda_{1}-1\right)}
$$




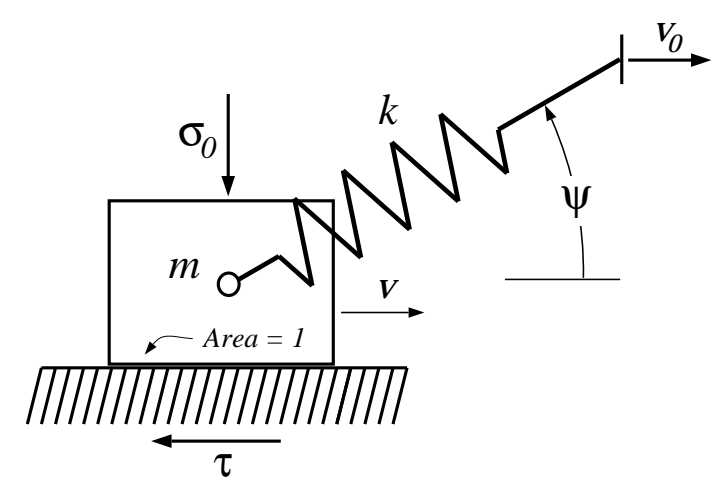

Figure 4: Inclined-spring model.

and

$$
\begin{aligned}
k_{c r_{2}}= & -\frac{B_{1} f_{1}\left\{f_{1}\left(B_{3}+B_{1} \lambda_{1} r\right)+f r\left(\lambda_{1}-\lambda\right)+\lambda_{1} r^{2}\left(m+B_{0} f_{1} \lambda_{1}\right)\right\}}{f\left(1+f_{1} B_{2}\right)+r f_{1}\left(\lambda_{1}-1\right)\left(B_{0}-m B_{2}\right)+B_{1} B_{2} f_{1}^{2}} \\
& +\frac{r f \lambda(f+m r)-B_{3}\left(f f_{1}-m r f_{1}\left(\lambda_{1}-1\right)\right)+B_{0} f f_{1} \lambda \lambda_{1} r^{2}}{f\left(1+f_{1} B_{2}\right)+r f_{1}\left(\lambda_{1}-1\right)\left(B_{0}-m B_{2}\right)+B_{1} B_{2} f_{1}^{2}}
\end{aligned}
$$

The first root can be discarded since the corresponding frequency is imaginary.

$$
\beta_{1}^{2}=-r^{2}
$$

Therefore, the required critical stiffness is $k_{c r_{2}}$. For a particular case, $(20)$ can be simplified and expressed in terms of the parameters of the state variable model and those of (12). This will be exemplified through the inclined-spring system discussed in the rest of the paper.

\section{Inclined-Spring Model}

Consider a mass of unit base area, pulled by a spring of stiffness $k$ at an angle $\psi$ as shown in Figure 4. This system illustrates normal stress coupling to displacement through stiffness. For example, the slider of a slider-crank mechanism can be modeled in this way. Dieterich and Linker (1992) analyzed this system in connection with fault slip and the triaxial test.

The normal stress, $\sigma$, is given by

$$
\sigma(t)=\sigma_{s s}+\sigma_{\Delta}(t)
$$

where

$$
\sigma_{\Delta}=k x \tan \psi
$$

As before, $x$ represents the shortening of the spring from the its steady-state length. 
Comparing its Laplace transform with the general form of normal stress proposed in (12),

$$
B_{2}=\tan \psi \quad B_{0}=B_{1}=B_{3}=B_{4}=0
$$

As will be shown in the next section, due to the magnitude of the velocities under consideration, the inertial forces can sometimes be neglected. The critical stiffness for the resulting quasistatic model is obtained from the inertial expression by setting the mass to zero.

For the state variable law expressed by (1), the critical stiffnesses are

$$
k_{c r}= \begin{cases}\frac{\frac{\sigma_{s s}(B-A)}{L}\left(\frac{A \sigma_{s s}}{v_{0}}+\frac{m v_{0}}{L}\right)}{\left\{1+\tan \psi\left(\mu_{s s}-\alpha\right)\right\} \frac{A \sigma_{s s}}{v_{o}}-\alpha \tan \psi \frac{m v_{0}}{L},} & \text { dynamic } \\ \frac{\sigma_{s s}(B-A)}{L\left\{1+\left(\mu_{s s}-\alpha\right) \tan \psi\right\}}, & \text { quasistatic }\end{cases}
$$

It is interesting to note that, in the quasistatic case, the critical stiffness reduces to that of constant normal stress when either $\psi=0$ or $\mu_{s s}=\alpha$. The latter case occurs when there is no instantaneous dependence of friction on normal stress.

To validate the results for critical stiffness, the nonlinear quasistatic and inertial models of the inclined-spring system were simulated. The dynamic equations are derived in (Bapna 1992). The following parameter values, taken from (Linker and Dieterich 1992), were used.

$$
\begin{array}{lll}
A=0.0145, & B=0.0160, & L=10^{-6} \mathrm{~m} \\
\sigma_{0}=5 \times 10^{6} \mathrm{~N} / \mathrm{m}^{2}, & \mu_{s s}=0.7, & \alpha=0.56
\end{array}
$$

Figures 5 shows the effect of stiffness for the quasistatic model with $\psi=-\pi / 4$. The solid line in this figure is the loci of steady-state points. The case depicted corresponds to a load-point velocity of $v_{0}$. The closed trajectory in the figure occurs for $k=k_{c r}$. The system is unstable for $k<k_{c r}$ and stable for $k>k_{c r}$.

To examine the effect of spring angle on system performance, a single value of stiffness, $k>k_{c r}(\psi=-\pi / 4)$ is used in Figure 6 . Negative spring angles cause the system to converge more rapidly to the equilibrium point. The critical stiffness is, however, smallest for positive spring angles.

\subsection{Lock-up Condition}

For the quasistatic case of the inclined-spring system, (18) and (25) yield

$$
\beta_{2}^{2}=\frac{(B-A)}{A}\left(\frac{v_{0}}{L}\right)^{2}\left(\frac{1+\mu_{s s} \tan \psi}{1+\left(\mu_{s s}-\alpha\right) \tan \psi}\right)
$$




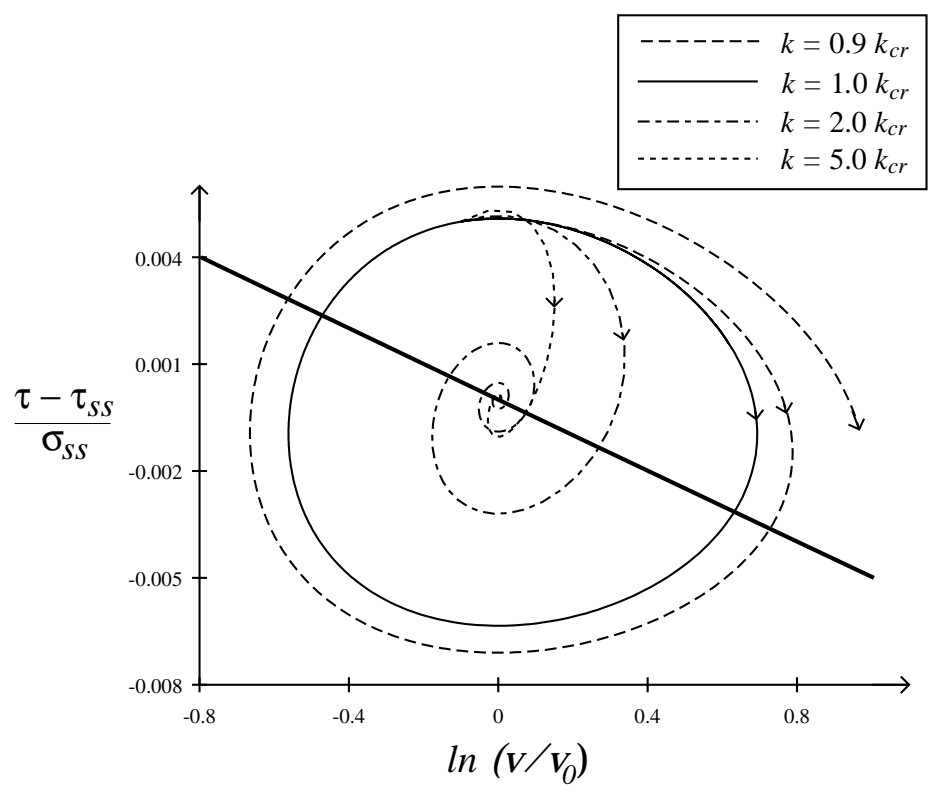

Figure 5: Effect of spring stiffness with $\psi=-\pi / 4$.

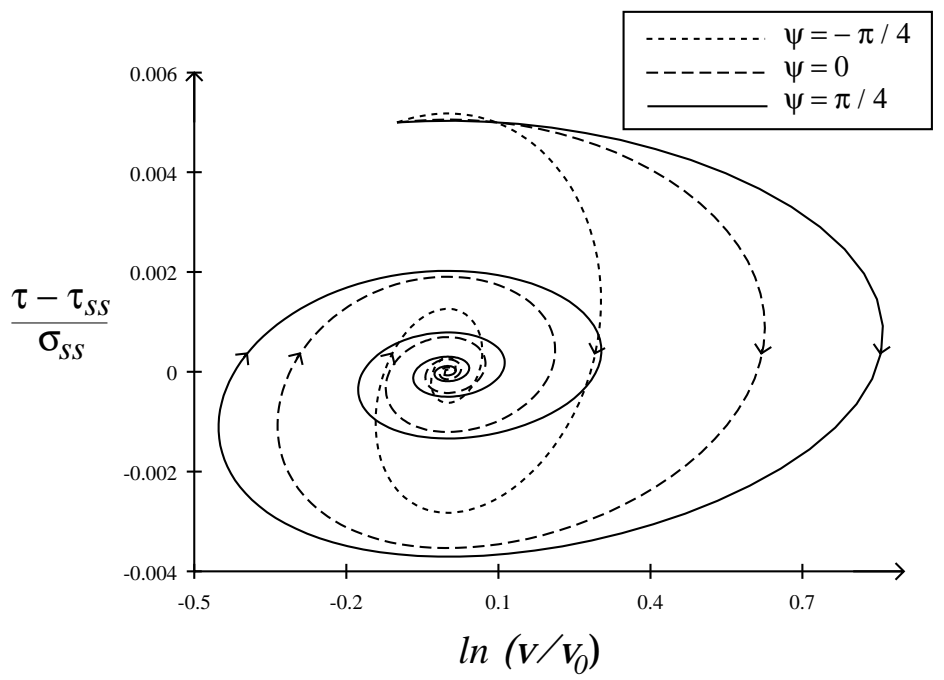

Figure 6: Comparison of trajectories for various $\psi$ with fixed $k>k_{c r}(\psi=-\pi / 4)$. 
This expression is positive for $\psi>0$, i.e., when the spring is pulling the block out of the surface. It can only be negative when $\left(1+\left(\mu_{s s}-\alpha\right) \tan \psi\right)>0$ and $\left(1+\mu_{s s} \tan \psi\right)<0$. The latter inequality, which also applies to the inertial model, corresponds to a spring angle within the friction cone defined by $\mu_{s s}$. A necessary condition for steady motion is that the horizontal component of the spring force be at least as large as the steady-state friction stress that the spring generates. This condition can be expressed as

$$
\psi>-\cot ^{-1}\left(\mu_{s s}\right)
$$

\subsection{Quasistatic Approximation}

Some insight about the range of masses for which the quasistatic model provides a close approximation of system behavior can be obtained by considering the critical stiffness. The dimensionless critical stiffness, $\kappa_{c r}=k_{c r} L / \sigma_{s s}$, for the inertial system is given by

$$
\kappa_{c r}=\frac{(B-A)\left(A+m_{*}\right)}{A\left[1+\tan \psi\left(\mu_{s s}-\alpha\right)\right]-\tan \psi m_{*} \alpha}
$$

where dimensionless mass is given by $m_{*}=m v_{0}^{2} / \sigma_{s s} L$.

The critical stiffnesses of the quasistatic and inertial models are nearly equal for

$$
m_{*}<<A=0.0145
$$

Simulation was used to verify this condition. The approximation is best when the spring is pulling the block into the surface.

The actual mass, $m$, expressed in terms of the dimensionless mass, $m_{*}$, is

$$
m=\frac{m_{\star} \sigma_{s s} L}{v_{0}^{2}}
$$

From this expression, it is clear that even $m_{*}=0.001$ represents a large mass when considering moderate normal stresses and low velocities. Thus, even with relatively large masses, the quasistatic model may be a good approximation when considering perturbations about the equilibrium point.

\subsection{Inertial Effects}

As seen in Figure 7, the effect of mass is to increase the critical stiffness and thus, to destabilize the system.

For the quasistatic case, the effect of increasing the spring angle, $\psi$, was to decrease the critical stiffness. Figure 7 shows, however, that after certain value of mass, labeled $m_{* c r}$, 

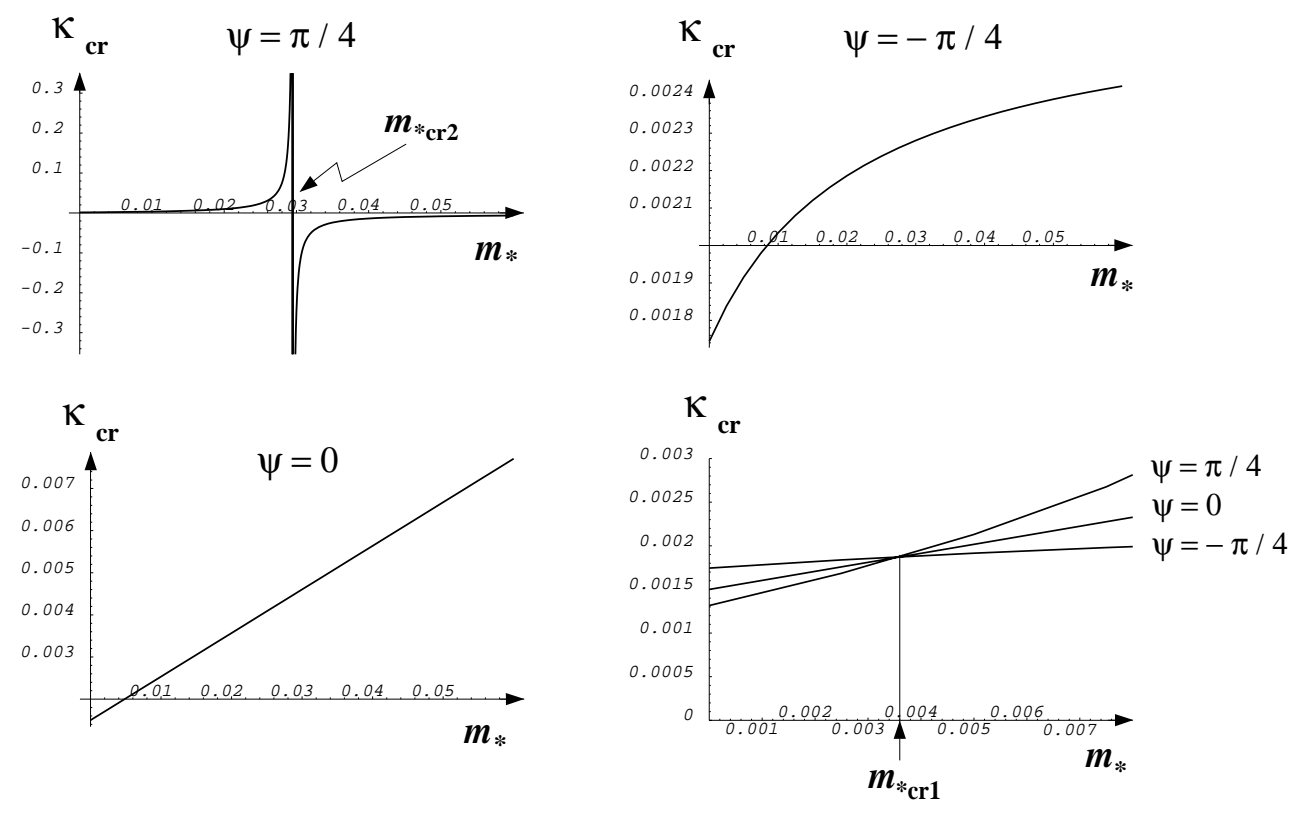

Figure 7: Critical stiffness, $\kappa_{c r}$, as a function of dimensionless mass, $m_{*}$.

this effect is reversed. This critical mass, at which $k_{c r}$ is independent of $\psi$, is given by

$$
m_{* c r 1}=\frac{A\left(\mu_{s s}-\alpha\right)}{\alpha}
$$

For this choice of parameters, $m_{* c r 1}=0.0036=0.25 \mathrm{~A}$. Since $0 \leq \alpha \leq \mu_{s s}$, it follows that $0 \leq m_{* c r 1} \leq+\infty$. This indicates that the quasistatic model must be used with caution. For large $\alpha / \mu_{s s}$, the reversal of $\kappa_{c r}$ 's dependence on spring angle occurs for small $m_{*}$. Even though the quasistatic criterion might be met, any comparisons based on spring angle using the quasistatic model could be invalid.

As seen in Figure 7, the critical stiffness, (28), possesses a singularity for positive spring angles. At this point, labeled $m_{* c r 2}, \beta_{2}^{2}$ becomes negative. For positive spring angles, no finite stiffness can stabilize the system for $m_{*} \geq m_{* c r 2}$. Note that $m_{* c r 2}>m_{* c r 1}$.

$$
m_{* c r 2}=\frac{A\left[1+B_{2}\left(\mu_{s s}-\alpha\right)\right]}{\alpha B_{2}}=m_{* c r 1}+\frac{A}{\alpha \tan \psi}
$$

The effect of increasing the spring angle is to decrease $m_{* c r 2}$. For a given $m_{*}>m_{* c r 1}$, a maximum spring angle exists above which the system is unstable. This situation does not correspond to the block losing contact with the surface since the normal stress is given by

$$
\sigma=\sigma_{s s}(1+\kappa x / L \tan \psi)
$$

Clearly, loss of contact can occur for any spring angle given appropriate initial conditions.

Note that both critical masses approach infinity as $\alpha$ goes to zero. Thus, their effects will only be seen in systems for which friction lags changes in normal stress. 


\section{Conclusions}

Experimental observations indicate that frictional stress responds to changes in normal stress with both instantaneous and evolutionary components. Since normal stress is not constant during the motion of most systems, but rather is a function of system state, it is important to gain an understanding of its effect and importance.

In this paper, the stability of a system with a single mechanical degree of freedom was studied subject to a state variable friction law proposed by Linker and Dieterich (1992). While this law is based on certain assumptions which may not hold for all systems, it is perhaps the simplest law to incorporate all effects relating to history dependence and may provide a qualitative indication of the behavior of more general friction laws.

An expression for the critical stiffness of the linearized model was derived for constant loadpoint velocity and normal stress dependent on slip displacement, velocity and acceleration. This general expression for normal stress covers a large class of systems and provides a lower bound on the combined machine/controller stiffness for stable motion.

The inclined-spring model was used as an example of a system for which normal stress is coupled through system stiffness to displacement. Two constraints on system mass were identified which indicate when the quasistatic model can be expected to provide a good approximation of the inertial system. When these constraints are met, the critical stiffness is smaller for positive spring angles $(\psi>0)$ than for negative angles. However, systems with positive spring angles exhibit less damping when perturbed from steady sliding.

It was also shown that, for steady sliding, there exist both upper and lower bounds on spring angle. For negative spring angles, the bound is the same for the quasistatic and inertial models and is the lock-up condition associated with the steady-state friction cone. For positive spring angles, a new type of bound was identified which is observed only for systems of sufficient mass and only when friction depends on normal stress history.

This analysis indicates that it is important to take the effect of varying normal stress into account. The value of system stiffness necessary to maintain stable sliding motion can depend quite significantly on normal stress and its coupling to system state. The normal stress can produce either a stabilizing or destabilizing effect on the system depending on the coupling and system parameters. These results may be important for machine design and control.

\section{References}

J. Anderson and A. Ferri 1990, "Behavior of a Single-Degree-of-Freedom System with a Generalized Friction Law." Journal of Sound and Vibration, Vol. 140, No. 2, pp. 287-304.

B. Armstrong-Hélouvry 1991, Control of Machines with Friction. Norwell, MA: Kluwer Academic Press. 
D. Bapna 1992, “The Stability of Sliding Systems with Friction Subject to Varying Normal Stress." M.S. Thesis, Aerospace and Mechanical Engineering, Boston University.

A. Bindemann and A. Ferri 1992, "Characteristics of Passive Damping in Built-up Structures." Friction-Induced Vibration, Chatter, Squeal and Chaos, ASME Winter Annual Meeting, Anaheim, CA, DE-Vol. 49, pp. 173-182.

J. Dieterich 1979, "Modeling of Rock Friction: 1. Experimental Results and Constitutive Equations." Journal of Geophysical Research, Vol. 84, pp. 2161-2168.

J. Dieterich 1991, "Micro-mechanics of Slip Instabilities with Rate- and State-dependent Friction." (Abstract), Eos, Trans. Am. Geophys. Union, Fall Meeting Abstract Volume, pp. 324.

J. Dieterich and M.F. Linker 1992, "Fault Stability Under Conditions of Variable Normal Stress." Geophysical Research Letters, Vol. 19, No. 16, pp. 1691-1694.

P. Dupont 1994, “Avoiding Stick-slip Through PD Control." IEEE Transactions on Automatic Control, to appear.

P. Dupont and E. Dunlap 1993, "Friction Modeling and Control in Boundary Lubrication." Proc. 1993 American Control Conference, San Francisco, CA, June, pp. 1910-1914.

J. Gu, J. Rice, A. Ruina and S. Tse 1984, "Slip Motion and Stability of a Single Degree of Freedom Elastic System with Rate and State Dependent Friction." J. Mech. Phys. Solids, Vol. 32, No. 3, pp. 167-96.

B. Hobbs and B. Brady 1985, "Normal Stress Changes and the Constitutive Law for Rock Friction." (abstract), Eos Trans. AGU, Vol. 66, pp. 382.

M. Linker and J. Dieterich 1992, "Effects of Variable Normal Stress on Rock Friction: Observations and Constitutive Equations." Journal of Geophysical Research, Vol. 97(B4), April, pp. $4923-4940$.

D. Lockner, R.Summer, and J. Byerlee 1986, "Effects of Temperature and Sliding Rate on Frictional Strength of Granite." Pure Appl. Geophys., Vol. 124, pp. 445-485.

E. Rabinowicz 1958, "The Intrinsic Variables Affecting the Stick-slip Process." Proc. Phys. Soc. London, Vol. 71, pp. 668-675.

J. Rice and A. Ruina 1983, "Stability of Steady Frictional Slipping." Journal of Applied Mechanics, Vol. 50, June, pp. 343-349.

A. Ruina 1983, "Slip Instability and State Variable Laws." Journal of Geophysical Research, Vol. 88, December, pp. 10359-10370. 